\title{
Revisión
}

\section{Explorando el poder de los abordajes transcriptómicos para identificar biomarcadores asociados a daño renal en pacientes con lupus eritematoso sistémico}

\author{
Valentina Arrieta Bravo (D)1, Tiffany Rangel Gómez ${ }^{\text {ID }}{ }^{1}$ y Lisandro Pacheco \\ Lugo (D口 $\square^{2}$ \\ ${ }^{1}$ Programa de Medicina, Facultad de Medicina, Universidad Simón Bolívar, Barranquilla, Colombia. \\ ${ }^{2}$ Grupo de investigación en Genética, Facultad de Ciencias Básicas y Biomédicas, Universidad Simón Bolívar, \\ Barranquilla, Colombia.
}

Recibido: 10/Jun/2020 Aceptado: 28/Jul/2020

Publicado: 02/Feb/2021
Cómo citar: Arrieta Bravo V, Rangel-Gómez T, Pacheco-Lugo L. Explorando el poder de los abordajes transcriptómicos para identificar biomarcadores asociados a daño renal en pacientes con lupus eritematoso sistémico. Rev. Colomb. Nefrol. 2021; 8(1), e492. https:/doi.org/10.22265/ acnef.8.1.492

\section{Resumen}

El lupus eritematoso sistémico (LES) es una enfermedad compleja y altamente heterogénea que afecta múltiples órganos, incluyendo articulaciones, corazón, sistema hematopoyético, sistema nervioso y riñón, siendo este último el de peor pronóstico y el que conlleva a nefritis lúpica (NL). Aunque la etiopatogénesis del LES aún no se conoce con claridad, se cree que la susceptibilidad genética y las modificaciones epigenéticas aberrantes favorecen su desenlace. Para establecer una terapia precisa es necesario evaluar de manera eficiente y objetiva el compromiso de órganos y la actividad de la enfermedad, lo cual es muy difícil por las pocas pruebas de laboratorio clínico disponibles en la actualidad.

En las últimas décadas la búsqueda de nuevos biomarcadores de LES ha sido una tendencia y se han identificado varios promisorios a nivel de genómica, metabolómica, proteómica y transcriptómica. En esta revisión se resume el estado del arte relacionado con estudios transcriptómicos que han identificado diversos transcritos potencialmente útiles como biomarcadores del LES y la NL.

Palabras clave: lupus eritematoso sistémico, nefritis lúpica, biomarcadores, microARNs.

$\square$ Correspondencia: Lisandro Pacheco, Facultad de Ciencias Básicas y Biomédicas, Carrera 59 No. 59-65, Universidad Simón Bolívar, Barranquilla, Colombia. Correo-e: lpacheco28@unisimonbolivar.edu.co 


\title{
Exploring the power of transcriptomic approaches to identify biomarkers associated to renal damage in Systemic Lupus Erythematosus patients
}

\begin{abstract}
Systemic Lupus Erythematosus is a complex and highly heterogeneous disease affecting multiple organs such as joints, heart, hematopoietic system, nervous system and kidney, the latter being the worst prognosis and leading to lupus nephritis (LN). While the etiopathogenesis of SLE is still not completely clear, is believed that genetic susceptibility and aberrant epigenetic modifications favor the outcome of the disease. In order to establish an accurate therapy, it is necessary to efficiently and objectively assess organ involvement and disease activity, which is very difficult due to the clinical laboratory tests currently available. In recent decades, the search for new SLE biomarkers has been a trend and many promising biomarkers have been identified at the genomic, metabolomic, proteomic and transcriptomic levels. In this review we summarize the state of the art related to transcriptomic studies that have identified various potentially useful transcripts as biomarkers of SLE and NL.
\end{abstract}

Keywords: Systemic lupus erythematosus, lupus nephritis, biomarkers, microRNAs.

\section{Introducción}

La autoinmunidad se refiere a las anormalidades en la actividad y la función del sistema inmune que llevan a efectos deletéreos de una pérdida de tolerancia a autoantígenos. La respuesta inmune puede manifestarse a través de disfunción multiorgánica y desencadenar en enfermedades sistémicas como el lupus eritematoso sistémico (LES), que se caracteriza por la presencia de autoanticuerpos dirigidos contra antígenos nucleares que forman inmunocomplejos (IC), principalmente depositados en los órganos afectados [1].

Entre las manifestaciones orgánicas del LES se encuentra el compromiso renal definido con nefritis lúpica (NL); este es el más temido, ya que incrementa de manera significativa la morbimortalidad. La NL es una glomerulonefritis mediada por IC que afecta a más del $60 \%$ de los pacientes con LES y se considera una causa importante de enfermedad renal crónica $(\mathrm{ERC})[2-4]$.

La incidencia y la severidad del compromiso renal varían entre individuos con LES, pero parecen estar asociadas a la etnicidad, ya que los afroamericanos, asiáticos e hispanos son más propensos a desarrollar enfermedad renal en comparación con pacientes euroamericanos [5]. 
A pesar de la disponibilidad de inmunoterapias agresivas como la ciclofosfamida y el micofenolato de mofetilo [6], la ERC en pacientes con LES finaliza en un pobre desenlace clínico, pues estos medicamentos producen una vasta gama de efectos colaterales [7]. Por lo tanto, hay una necesidad constante y crítica de elucidar los mecanismos inmunes detrás de la NL para poder identificar nuevos biomarcadores que sean fácilmente evaluables y faciliten el diagnóstico y monitoreo de los pacientes, lo cual llevará al desarrollo de terapias mucho más efectivas con mejores resultados y menos efectos adversos.

En la presente revisión se exploró el estado del arte relacionado con estudios cuyo foco fuera el descubrimiento de nuevos marcadores de NL en individuos con LES usando abordajes transcriptómicos. Se entendió como transcriptoma a todos los transcritos que se producen en una célula bajo cierta condición fisiológica y que son clave para la compresión del efecto de la actividad biológica de un grupo de genes en el contexto de salud/enfermedad [8]. Por su parte, la transcripción se entendió como un proceso nuclear cuya activación depende de estímulos intra o extracelulares que activan cascadas de señalización para determinar cuáles genes deben expresarse o reprimirse según el tipo de estímulo inicial [8].

\section{Expresión diferencial de factores de transcripción en el LES y la NL}

Diversos estudios han sugerido que existe una correlación marcada entre el LES y la expresión diferencial de diversos factores de transcripción (FT) [9-13] que juegan un papel central en la regulación de la expresión génica [14], por lo que alteraciones en su regulación, estructura y función podrían tener un impacto importante en el desarrollo y función del sistema inmune.

Sui et al. [15] realizaron un estudio con pacientes con LES y controles sanos en células mononucleares de sangre periférica (CMSP) cuyo objetivo principal era identificar FT diferencialmente expresos durante el LES. En dicho estudio se detectaron 92 de estos factores expresados diferencialmente: 78 regulados de forma aumentada y 14

\section{Estudios cuyo foco fuera el} descubrimiento de nuevos marcadores de NL en individuos con LES usando abordajes transcriptómicos

regulados de forma negativa en individuos con LES en comparación con los controles sanos. Entre esos FT expresos diferencialmente, se encontró que la proteína activadora 1 (AP-1), el Pbx1 y el factor potenciador de miocitos 2 (MEF-2) estaban asociados a la patogénesis y eran potenciales biomarcadores de diagnóstico [15]. 
Los FT FOXO forhhead juegan un papel importante en el control de la activación y proliferación de los linfocitos. Al respecto, Kuo \& Lin [16] realizaron un estudio en CMSP evaluando la desregulación de la expresión transcripcional de FOXO en pacientes con LES y artritis reumatoide (AR) en comparación con controles sanos, y encontraron que los niveles de transcripción de FOXO1 en pacientes con LES y AR activa fueron significativamente más bajos que los de controles sanos, relacionado posiblemente con la patogénesis [16].

Las células T activadas, las células B y la secreción potenciada de citocinas proinflamatorias pueden dañar los tejidos y órganos. Varias citocinas proinflamatorias, tales como IL-4, IFNg, IL-10, IL-17 y TGF-b, juegan funciones importantes durante el LES. T-bet y GATA-3 son los principales FT que conducen la diferenciación de los linfocitos Th1 y Th2, respectivamente.

Sui et al. [14] encontraron niveles aumentados de ARNm de T-bet e IFN- $\gamma$ y niveles disminuidos de ARNm de GATA-3 e IL-4 en pacientes con LES, lo que se ha correlacionado con enfermedad activa; estos autores también relacionaron el halotipo de riesgo de STAT-4 que tiene su función en la señalización del interferón tipo I y que se sobreexpresa por acción de algunos FT, incluidos AP-1 y NF-kB, y encontraron que el TF-IR5 es un mediador importante de la expresión activada por el receptor Toll-like de citocinas proinflamatorias tales como IFN tipo I y TNF- $\alpha$, que además está relacionado con la expresión del gen STAT-4. Asimismo, Frangou et al. [17] establecieron que la expresión de genes dirigidos por IRF-5 y STAT-4 podría distinguir a los pacientes con LES de individuos sanos.

\section{MicroARN (miARN) y su papel en el LES y la NL}

La epigenética es el estudio de los cambios reversibles y potencialmente heredables en la expresión génica sin alteraciones del código genético. Entre las principales modificaciones epigenéticas se encuentra la metilación del ADN, la modificación de histonas y los miARN [18], que son secuencias cortas de ARN no codificantes que regulan la expresión génica al bloquear la traducción de proteínas o inducir la degradación de ARNm [19]. Diferentes estudios han abordado el papel de los miARN como biomarcadores diagnósticos del LES y complicaciones frecuentes como la NL.

En relación con la epigenética asociada al LES, para desentrañar un papel potencial de los microARN en la hipometilación aberrante del ADN en el LES se realizó un perfil de microARN en las células TCD4+ de pacientes con la enfermedad y ratones con tendencia al lupus. Asimismo, se descubrió que tres microARN sobreregulados (miR-21, miR-148a y miR-126) promovían la hipometilación de las células TCD4+. Además, las modificaciones de histonas 
y la metilación del ADN en regiones reguladoras de la secuencia miR-142 disminuyen su expresión, lo que contribuye a la activación de células TCD4+ y a la hiperestimulación de células B en LES [17]. Este mismo estudio reportó que un grupo de miARN (miR-25, miR-106b y miR-21) se encontraba con expresión aumentada tanto en células $\mathrm{B}$ como en células $\mathrm{T}$ de individuos con LES, y que estos tuvieron la correlación más fuerte con enfermedad activa [17]. En otro estudio, Stagakis et al. [20] encontraron que el miR-21 regulado negativamente afecta la expresión de PDCD4, un inhibidor selectivo de la traducción de proteínas de genes involucrados en las respuestas inmunes que regula las respuestas aberrantes de células $\mathrm{T}$ en el LES humano, lo que podría representar un biomarcador de enfermedad y un objetivo terapéutico en esta enfermedad.

El grupo de Nefrología de la Universidad Simón Bolívar viene trabajando activamente en la búsqueda de miARN como biomarcadores de daño renal en pacientes con LES: en un estudio observacional en el que se caracterizaron los perfiles de expresión diferencial de miARN en pacientes con LES con diferentes grados de NL y se compararon con pacientes con LES sin afectación renal e individuos de control sanos, Navarro-Quiroz et al. [21] encontraron cinco microARN (miR-221-5p, miR-380-3p, miR-556-5p, miR-758-3p y miR-3074-3p) que en su conjunto permiten identificar, con altos valores de sensibilidad y especificidad y con valores predictivos positivos y negativos, individuos con afectación renal y LES. Estos resultados

La epigenética es el estudio de los cambios reversibles y potencialmente heredables en la expresión génica sin alteraciones del código genético sugieren que dichas moléculas circulantes en plasma pueden ser potenciales biomarcadores diagnósticos de la NL en pacientes con LES. Es importante mencionar que el patrón diferencial observado en la abundancia de miARN puede tener implicaciones funcionales en la fisiopatología del daño renal por LES.

En otro trabajo, cuyo objetivo era identificar in silico las relaciones entre los microARN y los genes que codifican FT, ubiquitinación, metilación del ADN y modificaciones de histonas en el LES, Navarro et al. [22] identificaron 226 miARNs con expresión diferencial. De manera interesante, Navarro et al. [23] también observaron que alteraciones de miARN como hsa-miR-30a-5p, hsa-miR-16-5p, hsa-miR-142-5p y hsa-miR-324-3p están más asociadas a modificaciones postraduccionales, mientras que hsa-miR-16-5p, hsa-miR-374a-5p, hsa-miR34a- $5 p$, hsa-miR-31-5p y hsa -miR-1-3p se asocian con mayor frecuencia a genes relacionados con la susceptibilidad del LES; en este mismo trabajo se reportaron 24 miARN circulantes en plasma con abundancia diferencial, 14 descritos por primera vez en NL (hsa-miR-589-3p, 
hsa-miR-1260b, hsa-miR-4511, hsa-miR-485- 5p, hsa-miR-584-5p, hsa-miR-543, hsa-miR-1533p, hsa-miR-6087, hsa-miR-3942- 5p, hsa-miR-7977, hsa-miR-323b-3p, hsa-miR-4732-3p y hsa-miR-6741-3p).

Estos cambios en la abundancia de miARN podrían interpretarse como alteraciones en la red reguladora de miARN-mARN en la patogénesis de la NL antes del inicio clínico de la enfermedad, por lo tanto contribuyen a comprender el proceso de la enfermedad y es probable que allanen el camino hacia la identificación de biomarcadores para el diagnóstico temprano de LES.

Martínez-Ramos et al. [24], usando TaqMan Human MicroRNA Array, Identificaron 377 miARN en células B CD19+ y células T CD4+, de los cuales cuatro (hsa-miR-143, hsa-miR-224, hsa-miR-10a y hsa-miR-345) tuvieron expresión aumentada en estas poblaciones celulares en individuos con LES respecto a individuos clínicamente sanos. En otro estudio, Kusaoi et al. [25] reportaron que los pacientes con LES presentan patrones de expresión distintos de miARN según la progresión de la enfermedad, además encontraron ocho miARN (hsa miR 494, hsa miR 188, hsa miR 501, mmu miR 298, HMP PREDICTED MIR61, HMP PREDICTED MIR78, hsa miR 296 y hsa miR 299 3p) que mostraron un patrón de expresión diferencial aumentada en pacientes con LES con enfermedad activa al compararlos con individuos con LES inactivo, lo que permite hipotetizar que estos miARN pueden estar involucrados en la progresión de la enfermedad y el daño de órganos.

Por otra parte, Jafari-Ghods et al. [26] aislaron ARNm y miARN totales de muestras de sangre para ser usados en ensayos de microarreglos de ADN. Ellos describieron dos miARN (hsa-miR-766-3p y has-mir-5571-5p) con expresión significativamente reducida en sujetos con LES y afectación renal en relación a los individuos con LES y sin afectación renal. De manera interesante, los investigadores encontraron que el hsa-miR-766-3p puede jugar un rol trascendental en la vía PI3K-AKT-mTOR [26].

Te et al. [27] identificaron cinco miARN (hsa-miR-371-5P, hsa-miR-423-5P, hsa-miR- 638, hsa-miR-1224-3P y hsa-miR-663) expresados diferencialmente en distintos grupos raciales (afroamericanos, europeos y americanos) asociados a NL, aunque solo el hsa-miR-342-3P se expresó diferencialmente en muestras de sujetos afroamericanos. Carlsen et al. [28], en un estudio con 409 muestras de plasma de 364 participantes (pacientes con LES, sujetos de control sanos y sujetos de control con otras enfermedades autoinmunes) reportaron que cuatro miARN (miR-142-3p, miR- 106a, miR-17 y miR-20a) pueden ser biomarcadores confiables de LES y que un subconjunto específico de perfiles de miARN se asoció con NL. 
Todos los miARN característicos se dirigen a genes en las vías de señalización del factor de crecimiento transformante $\beta$. Otras dianas moleculares incluyen la regulación de la apoptosis, los receptores de citocinas, el desarrollo de células T y la organización del citoesqueleto. Estos hallazgos resaltan posibles vías desreguladas en el LES y sugieren que los patrones de miARN circulantes distinguen al LES de otros fenotipos inmunoinflamatorios.

Se ha reportado que la expresión intrarrenal de ciertos miARN tiene relación con la actividad de la enfermedad [29-31]. Por ejemplo, Krasoudaki et al. [32] concluyeron que, en comparación con el tejido normal renal, un grupo de 24 miARN define la NL humana: nueve mostrando expresión aumentada y 15 mostrando expresión disminuida. Entre estos, el miR-422a exhibió la más alta regulación, cuya diana pareció ser la calicreína 4 (KLK4), una serina esterasa secretada con propiedades de remodelación de la matriz extracelular y angiogénica en la patogénesis de la NL. La familia de genes de calicreína tiene un papel importante en la regulación de la inflamación, apoptosis, coagulación y fibrosis en los riñones.

Sui et al. [15] compararon la expresión de miARN en biopsias renales de pacientes con NL y controles normales, e identificaron 30 miARN regulados negativamente y 36 con expresión aumentada. En otro estudio realizado por Carlsen et al. [28], siete miARN se expresaron estadísticamente de manera diferencial en plasma de pacientes con LES y la expresión de miR142-3p y miR-181a aumentó, mientras que la de miR-106a, miR-17, miR-20a, miR-203 y miR-92 ${ }^{a}$ disminuyó. Además, la expresión de miR-342-3p, miR-223 y miR-20a disminuyó significativamente en pacientes con LES y nefritis activa.

\section{MiARN, autoanticuerpos y sus implicaciones biológicas}

Trabajos anteriores han mostrado que es posible agrupar pacientes con LES según la especificidad de autoanticuerpos [33-36]. Rai et al. [33] identificaron patrones diferenciales de expresión de miARN en sujetos con LES con diferentes estados de autoanticuerpos y establecieron hipótesis acerca de sus implicaciones biológicas. En individuos con autoanticuerpos positivos para el antígeno nuclear extraíble (ENA, por su sigla en inglés para extractable nuclear antigen) (anti-ENA+), estos autores hallaron 28 miARN regulados negativamente. También encontraron que las vías que controlan el ciclo celular (regulación mediada por proteínas de la familia BTG, CHK y señalización de CDK5) y la remodelación del citoesqueleto (señalización de actina, de RhoGDI, de unión estrecha y la regulación de la motilidad de actina basada en Rho) estaban afectas en este subconjunto de pacientes. 
En los pacientes con autoanticuerpos contra dsDNA positivos (antidsDNA+) se hallaron 33 miARN regulados positivamente que, a su vez, regulan el control de varias vías de señalización de citocinas tales como IL-6, IL-17, IL-4, IL-2, CXCR4, CNTF y IL-10. Se encontraron también tres miARN regulados negativamente y sin información biológica relevante [31]. En cuanto a los sujetos con Anti-dsDNA + y anti- ENA +, se evidenció que los miARN asociados con las vías de respuesta viral y del huésped están desregulados en los subconjuntos.

Los pacientes con LES muestran firmas transcripcionales sanguíneas únicas relacionadas con el IFN tipo 1 y granulocitos, y se sugiere que estas firmas se pueden utilizar para evaluar la enfermedad activa. La mayoría de los estudios se han centrado en las transcripciones o proteínas inducidas por IFN como biomarcadores y sufren limitaciones de tamaño de la muestra y heterogeneidad clínica y terapéutica inherente a la enfermedad, lo que dificulta la interpretación de los datos [32].

\section{¿Podrían los miARN y ARN mensajeros circulantes en orina correlacionarse con enfermedad renal activa?}

Diferentes tipos de abordajes "ómicos" han explorado el potencial de la orina como biofluido ideal para el descubrimiento de nuevos biomarcadores del LES y la NL en particular, lo cual se debe básicamente a que la orina ofrece muchas ventajas sobre otros biofluidos, entre las que resaltan cuatro aspectos principales:

1. La concentración de proteínas, de pequeños metabolitos y de ciertos transcritos es más abundante en la orina que en plasma o suero.

2. La orina contiene muchos metabolitos que permiten predecir el desbalance de casi todas las rutas bioquímicas del cuerpo.

3. Comparado con el plasma, la orina es colectada de forma no invasiva.

4. La orina se considera como una biopsia no invasiva del riñón [37,38].

En ese sentido, la búsqueda del biomarcador ideal de daño renal en pacientes con LES podría tener la respuesta en la orina [39]. Cárdenas-González et al. [40] realizaron un perfil global de miARN urinarios e identificaron dos biomarcadores (miR-3201 y miR-1273e) que se correlacionaron con la NL; en los sujetos con NL ambos estaban regulados negativamente y se relacionaron con la presencia de inflamación glomerular endocapilar. Estos miARN permitieron discriminar con una fuerte asociación estadística entre pacientes con enfermedad renal versus sujetos sanos o con LES [40]. Asimismo, en el estudio de Abulaban et al. [41] se reportó que los niveles de miR-125a, miR-150 y miR-155 en orina están asociados con la 
expresión de biomarcadores de NL-Panel en niños, lo que sugiere un papel de estos en el diagnóstico de la NL en edad infantil.

Se ha propuesto que los miARN exosomales urinarios pueden reflejar de manera precisa la disfunción renal y el daño estructural en el riñón, haciendo de estas pequeñas moléculas excelentes dianas para la exploración de biomarcadores de enfermedades del tracto urinario [34, 42, 44, 45]. Solé et al. [46] reportaron que miR-21, $\mathrm{miR}-150, \mathrm{y} \operatorname{miR}-29 \mathrm{c}$ se correlacionaron con NL activa y podrían predecir un riesgo aumentado de progresión a ERC. Además, se ha reportado un aumento en los niveles de miR- 146a y miR-29c exosomal urinario en pacientes con NL activa y se sugiere que estos pueden servir como predictores de fibrosis renal temprana [18]. Otro estudio reciente encontró que la expresión de miR-3135b, miR-654-5p y miR-146a-5p en exosomas urinarios se correlaciona con NL clase IV con buena eficacia diagnóstica [47].

Guan et al. [48] realizaron un estudio en el que se midieron los niveles urinarios de miR-221, miR-222, miR-339-3P y miR-339-5P involucrados en la regulación de la molécula 1 de adhesión intercelular urinaria (ICAM-1), un potencial biomarcador de la NL y encontraron que los niveles de sedimento urinario de miR-339-3P están significativamente asociados con proteinuria, y que miR-221 y miR-222 están inversamente relacionados con el anti-dsDNA en suero. Además, miR-221 se correlacionó específicamente con los niveles séricos de C3.

En un análisis de transcriptoma de sedimento urinario de pacientes con LES, Jakiela et al. [49] reportaron que en pacientes con NL activa hubo una marcada regulación positiva de transcritos de respuesta inmune tipo I relacionados con células $\mathrm{T}$ (tales como CD3G) y, a la inversa, genes de respuesta inmune tipo II (tales como GATA3 y CCL17) significativamente reducidos. Estos resultados demuestran que una desregulación a nivel del control de la expresión génica tiene fuertes repercusiones en la evolución de la enfermedad renal.

\section{Conclusiones}

La búsqueda de un biomarcador que permita diagnosticar el compromiso renal en pacientes con LES en etapas tempranas es un campo activo de investigación, pues en la actualidad solo la biopsia renal puede mostrar con relativa sensibilidad el daño histológico. Sin embargo, por ser esta una técnica invasiva y por la imposibilidad de aplicarla de forma serial para seguimiento 
de pacientes con NL, se hace necesario seguir explorando la utilidad diagnóstica de nuevos marcadores en fluidos biológicos cuya recolección sea menos invasiva. En ese contexto, diversas moléculas producto de la regulación de la expresión génica a nivel del riñón surgen como potenciales dianas para diagnóstico. La Tabla 1 recoge información de diversos miARN que han sido encontrados en fluidos biológicos y que han mostrado una fuerte asociación con daño renal en pacientes con LES. Se prevé que la combinación de varios biomarcadores de LES y NL podrá direccionar de forma eficiente el tratamiento de estos pacientes.

Tabla 1. MicroARN identificados en diversos fluidos biológicos con potencial diagnóstico del lupus eritematoso sistémico

\begin{tabular}{|l|l|c|c|}
\hline \multicolumn{1}{|c|}{ MicroARN } & \multicolumn{1}{|c|}{ Fluido } & $\begin{array}{c}\text { correlación con } \\
\text { enfermedad } \\
\text { activa }\end{array}$ & Referencia \\
\hline $\begin{array}{l}\text { miR-221-5p, miR-380-3p, miR-556-5p, } \\
\text { miR-758-3p y miR-3074-3p }\end{array}$ & Suero & - & {$[21]$} \\
\hline miR-343-3p, miR-223 y miR-20 ${ }^{a}$ & Suero & - & {$[28]$} \\
\hline miR-221 y miR-222 & Orina & + & {$[48]$} \\
\hline miR-125a, miR-150 y miR-155 & Orina & - & {$[41]$} \\
\hline miR-146 ${ }^{a}$ & Exosoma urinario & + & {$[50]$} \\
\hline miR-29c & Exosoma urinario & - & {$[46]$} \\
\hline
\end{tabular}

Fuente: Elaboración con base en $\mathrm{Wu}$ et al. [18].

\section{Consideraciones éticas}

Los autores declaran que los procedimientos seguidos se realizaron conforme a las normas éticas del comité de experimentación humana responsable y de acuerdo con lo establecido por la Asociación Médica Mundial en la Declaración de Helsinki; que han seguido los protocolos de su centro de trabajo sobre la publicación de datos de pacientes, y que han obtenido el consentimiento informado de los pacientes y/o sujetos referidos en el artículo.

\section{Conflicto de intereses}

Ninguno declarado por los autores. 


\section{Financiación}

Esta revisión hace parte de un proyecto que viene siendo financiado por el Ministerio de Ciencia, Tecnología e Innovación, cuyo código es 125384467468.

\section{Contribución de los autores}

Valentina Arrieta Bravo: investigación, escritura (manuscrito original); Tiffany Rangel Gómez: investigación, escritura (manuscrito original); Lisandro Pacheco Lugo: conceptualización, investigación, escritura (manuscrito original), escritura (revisión y edición).

\section{Referencias}

[1] Honarpisheh M, Köhler P, von Rauchhaupt E, Lech M. The Involvement of MicroRNAs in Modulation of Innate and Adaptive Immunity in Systemic Lupus Erythematosus and Lupus Nephritis. J Immunol Res. 2018;2018:4123106. https://dx.doi.org/10.1155/2018/4126106 个Ver página 2

[2] Zhu H, Mi W, Luo H, Chen T, Liu S, Raman I, et al. Whole-genome transcription and DNA methylation analysis of peripheral blood mononuclear cells identified aberrant gene regulation pathways in systemic lupus erythematosus. Arthritis Res Ther. 2016;18(1):162. https:/dx.doi.org/10.1186/s13075-016-1050-x 个Ver página 2

[3] Koutsokeras T, Healy T. Systemic lupus erythematosus and lupus nephritis. Nat Rev Drug Discov. 2014;13(3):173-4. https://dx.doi.org/10.1038/nrd4227 个Ver página 2

[4] Arroyo AR, García R, Aroca G, Cadena A, Acosta J. Correlación clínica e inmunohistopatológica de la nefropatía lúpica en un centro de referencia del Caribe colombiano durante los años 2012 a 2013. Rev Colomb Nefrol. 2014;1(2):57-64. https://x.doi.org/10.22265/ acnef.1.2.176 $\uparrow$ Ver página 2

[5] Coit P, Renauer P, Jeffries MA, Merrill JT, McCune WJ, Maksimowicz- McKinnon K, et al. Renal involvement in lupus is characterized by unique DNA methylation changes in naïve CD4+ T cells. J Autoimmun. 2015;61:29-35. https://x.doi.org/10.1016/j.jaut.2015.05.003 $\uparrow$ Ver página 2

[6] Meliambro K, Campbell KN, Chung M. Therapy for Proliferative Lupus Nephritis. Rheum Dis Clin North Am. 2018;44(4):545-60. https:/dx.doi.org/10.1016/j.rdc.2018.06.002 个Ver página 3 
[7] Schwartz N, Goilav B, Putterman C. The pathogenesis, diagnosis and treatment of lupus nephritis. Curr Opin Rheumatol. 2014;26(5):502-9. https://dx.doi.org/10.1097/BOR. $0000000000000089 \uparrow$ Ver página 3

[8] Zumerle S, Alimonti A. In and out from senescence. Nat Cell Biol. 2020;22(7):753-4. https: //dx.doi.org/10.1038/s41556-020-0540-x 个Ver página 3

[9] Guo Y, Zhao M, Lu Q. Transcription factor RFX1 is ubiquitinated by E3 ligase STUB1 in systemic lupus erythematosus. Clin Immunol. 2016;169:1-7. https:/dx.doi.org/10.1016/j.clim. 2016.06.003 $\uparrow$ Ver página 3

[10] Luo J, Niu X, Liu H, Zhang M, Chen M, Deng S. Up-regulation of transcription factor Blimp1 in systemic lupus erythematosus. Mol Immunol. 2013;56(4):574-82. https:/dx.doi.org/ 10.1016/j.molimm.2013.05.241 个Ver página3

[11] Ban T, Sato GR, Tamura T. Regulation and role of the transcription factor IRF5 in innate immune responses and systemic lupus erythematosus. Int Immunol. 2018;30(11):529-36. https:/dx.doi.org/10.1093/intimm/dxy032 个Ver página 3

[12] Jiang T, Tian F, Zheng H, Whitman SA, Lin Y, Zhang Z, et al. Nrf2 suppresses lupus nephritis through inhibition of oxidative injury and the NF- $\kappa \mathrm{B}$-mediated inflammatory response. Kidney Int. 2014;85(2):333-43. https://dx.doi.org/10.1038/ki.2013.343 个Ver página 3

[13] Mathenia J, Reyes-Cortes E, Williams S, Molano I, Ruiz P, Watson DK, et al. Impact of Fli1 transcription factor on autoantibody and lupus nephritis in NZM2410 mice: Effect of Fli-1 gene on lupus in NZM2410 mice. Clin Exp Immunol. 2010;162(2):362-71. https://x.doi.org/ 10.1111/j1365-2249.2010.04245.x. 个Ver página 3

[14] Sui W, Hou X, Che W, Yang M, Dai Y. The applied basic research of systemic lupus erythematosus based on the biological omics. Genes Immun. 2013;14(3):133- 46. https:/dx.doi.org/ 10.1038/gene.2013.3 $\uparrow$ Ver página 3, 4

[15] Sui W, Lin H, Chen J, Ou M, Dai Y. Comprehensive analysis of transcription factor expression patterns in peripheral blood mononuclear cell of systemic lupus erythematosus. Int J Rheum Dis. 2012;15(2):212-9. https:/dx.doi.org/10.1111/j.1756-185X.2012.01718.x 个Ver página 3,7

[16] Kuo CC, Lin SC. Altered FOXO1 Transcript Levels in Peripheral Blood Mononuclear Cells of Systemic Lupus Erythematosus and Rheumatoid Arthritis Patients. Mol Med. 2007;13(11-

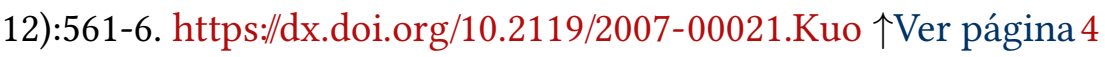


[17] Frangou EA, Bertsias GK, Boumpas DT. Gene expression and regulation in systemic lupus erythematosus. Eur J Clin Invest. 2013;43(10):1084-96. https://x.doi.org/10.1111/eci.12130 个Ver página 4,5

[18] Wu H, Zeng J, Yin J, Peng Q, Zhao M, Lu Q. Organ-specific biomarkers in lupus. Autoimmun Rev. 2017;16(4):391-7. https://dx.doi.org/10.1016/j.autrev.2017.02.011 $\uparrow$ Ver página4, 9, 10

[19] Li Y, Fang X, Li Q-Z. Biomarker Profiling for Lupus Nephritis. Genomics Proteomics Bioinformatics. 2013;11(3):158-65. https:/dx.doi.org/10.1016/j.gpb.2013.05.003 $\uparrow$ Ver página 4

[20] Stagakis E, Bertsias G, Verginis P, Nakou M, Hatziapostolou M, Kritikos H, et al. Identification of novel microRNA signatures linked to human lupus disease activity and pathogenesis: miR-21 regulates aberrant $\mathrm{T}$ cell responses through regulation of PDCD4 expression. Ann Rheum Dis. 2011;70(8):1496-506. https://dx.doi.org/10.1136/ard.2010.139857 $\uparrow$ Ver página 5

[21] Navarro-Quiroz E, Pacheco-Lugo L, Lorenzi H, Díaz-Olmos Y, Almendrales L, Rico E, et al. High-Throughput Sequencing Reveals Circulating miRNAs as Potential Biomarkers of Kidney Damage in Patients with Systemic Lupus Erythematosus. PLoS One. 2016;11(11):e0166202. https://dx.doi.org/10.1371/journal.pone.0166202 个Ver página 5, 10

[22] Navarro-Quiroz E, Navarro-Quiroz R, Pacheco-Lugo L, Aroca-Martínez G, GómezEscorcia L, González-Torres $\mathrm{H}$, et al. Integrated analysis of microRNA regulation and its interaction with mechanisms of epigenetic regulation in the etiology of systemic lupus erythematosus. PLoS One. 2019;14(6):e0218116. https://x.doi.org/10.1371/journal.pone.0218116 个Ver página 5

[23] Navarro-Quiroz E, Pacheco-Lugo L, Navarro-Quiroz R, Lorenzi H, España- Puccini P, Díaz-Olmos Y, et al. Profiling analysis of circulating microRNA in peripheral blood of patients with class IV lupus nephritis. PLoS One. 2017;12(11):e0187973. https://x.doi.org/ 10.1371/journal.pone.0187973 个Ver página 5

[24] Martínez-Ramos R, García-Lozano JR, Lucena JM, Castillo-Palma MJ, García- Hernández F, Rodríguez MC, et al. Differential expression pattern of microRNAs in CD4+ and CD19+ cells from asymptomatic patients with systemic lupus erythematosus. Lupus. 2014;23(4):353-9. https:/dx.doi.org/10.1177/0961203314522335 个Ver página 6

[25] Kusaoi M, Yamaji K, Ishibe Y, Murayama G, Nemoto T, Sekiya F, et al. Separation of Circulating MicroRNAs Using Apheresis in Patients With Systemic Lupus Erythematosus. Ther Apher Dial. 2016;20(4):348-53. https:/dx.doi.org/10.1111/1744-9987.12471 $\uparrow$ Ver página 6 
[26] Jafari-Ghods F, Topal-Sarikaya A, Arda N, Hamuryudan V. MiRNA and mRNA Profiling in Systemic Lupus Reveals a Novel Set of Cytokine - Related miRNAs and their Target Genes in Cases With and Without Renal Involvement. Kidney Blood Press Res. 2017;42(6):1322-37. https:/dx.doi.org/10.1159/000485987 个Ver página 6

[27] Te JL, Dozmorov IM, Guthridge JM, Nguyen KL, Cavett JW, Kelly JA, et al. Identification of unique microRNA signature associated with lupus nephritis. PloS One. 2010;5(5):e10344. https://dx.doi.org/10.1371/journal.pone.0010344 个Ver página 6

[28] Carlsen AL, Schetter AJ, Nielsen CT, Lood C, Knudsen S, Voss A, et al. Circulating MicroRNA Expression Profiles Associated With Systemic Lupus Erythematosus. Arthritis Rheum. 2013;65(5):1324-34. https:/dx.doi.org/10.1002/art.37890 个Ver página 6, 7

[29] Lu J, Kwan BC, Lai FM, Tam LS, Li EK, Chow K, et al. Glomerular and tubulointerstitial miR-638, miR-198 and miR-146a expression in lupus nephritis: miRNA in lupus nephritis. Nephrology (Carlton). 2012;17(4):346-51. https:/dx.doi.org/10.1111/j.1440-1797.2012.01573.x 个Ver página 7

[30] Rudnicki M, Perco P, D’haene B, Leierer J, Heinzel A, Mühlberger I, et al. Renal microRNA- and RNA-profiles in progressive chronic kidney disease. Eur J Clin Invest. 2016;46(3):213-26. https://dx.doi.org/10.1111/eci.12585 个Ver página 7

[31] Trionfini P, Benigni A, Remuzzi G. MicroRNAs in kidney physiology and disease. Nat Rev Nephrol. 2015;11(1):23-33. https:/dx.doi.org/10.1038/nrneph.2014.202 个Ver página 7, 8

[32] Krasoudaki E, Stagakis E, Loupasakis K, Papagianni A, Alexopoulos E, Bertsias G, et al. SAT0006 Microrna analysis of human lupus nephritis: Evidence for modulation of kallikrein 4 by MIR-422A. Ann Rheum Dis. 2013;71(Suppl 3):472- 3. https://x.doi.org/10.1136/ annrheumdis-2012-eular.2954 $\uparrow$ Ver página 7, 8

[33] Rai R, Chauhan SK, Singh VV, Rai M, Rai G. RNA-seq Analysis Reveals Unique Transcriptome Signatures in Systemic Lupus Erythematosus Patients with Distinct Autoantibody Specificities. PLoS One. 2016;11(11):e0166312. https://dx.doi.org/10.1371/journal.pone. $0166312 \uparrow$ Ver página 7

[34] Chauhan SK, Singh VV, Rai R, Rai M, Rai G. Distinct Autoantibody Profiles in Systemic Lupus Erythematosus Patients are Selectively Associated with TLR7 and TLR9 Upregulation. J Clin Immunol. 2013;33(5):954-64. https:/dx.doi.org/10.1007/s10875-013-9887-0 个Ver página 7,9

[35] Chauhan SK, Singh VV, Rai R, Rai M, Rai G. Differential microRNA Profile and PostTranscriptional Regulation Exist in Systemic Lupus Erythematosus Patients with Distinct 
Autoantibody Specificities. J Clin Immunol. 2014;34(4):491-503. https://x.doi.org/10.1007/ s10875-014-0008-5 个Ver página 7

[36] Rai R, Chauhan SK, Singh VV, Rai M, Rai G. Heat shock protein 27 and its regulatory molecules express differentially in SLE patients with distinct autoantibody profiles. Immunol Lett. 2015;164(1):25-32. https:/dx.doi.org/10.1016/j.imlet.2015.01.007 个Ver página 7

[37] Bramham K, Mistry HD, Poston L, Chappell LC, Thompson AJ. The non- invasive biopsywill urinary proteomics make the renal tissue biopsy redundant? QJM. 2009;102(8):523-38. https:/dx.doi.org/10.1093/qjmed/hcp071 个Ver página 8

[38] Magalhães P, Pejchinovski M, Markoska K, Banasik M, Klinger M, Švec-Billá D, et al. Association of kidney fibrosis with urinary peptides: a path towards non- invasive liquid biopsies? Sci Rep. 2017;7(1):16915. https://x.doi.org/10.1038/s41598-017-17083-w 个Ver página 8

[39] Pacheco-Lugo L, Díaz-Olmos Y, Aroca-Martínez G. Biomarcadores en fluídos biológicos y su potencial uso como indicadores de nefritis lúpica en individuos con lupus eritematoso sistémico. Rev Colomb Nefrol. 2014;1(1):39-47. https:/dx.doi.org/10.22265/acnef.1.1.171 $\uparrow$ Ver página 8

[40] Cárdenas-González M, Srivastava A, Pavkovic M, Bijol V, Rennke HG, Stillman IE, et al. Identification, Confirmation, and Replication of Novel Urinary MicroRNA Biomarkers in Lupus Nephritis and Diabetic Nephropathy. Clin Chem. 2017;63(9):1515-26. https://dx.doi. org/10.1373/clinchem.2017.274175 个Ver página 8

[41] Abulaban KM, Fall N, Nunna R, Ying J, Devarajan P, Grom A, et al. Relationship of cell-free urine MicroRNA with lupus nephritis in children. Pediatr Rheumatol Online J. 2016;14(1):4. https:/dx.doi.org/10.1186/s12969-016-0064-x 个Ver página 8, 10

[42] Santiago-Dieppa DR, Steinberg J, Gonda D, Cheung VJ, Carter BS, Chen CC. Extracellular vesicles as a platform for 'liquid biopsy' in glioblastoma patients. Expert Rev Mol Diagn. 2014;14(7):819-25. https://x.doi.org/10.1586/14737159.2014.943193 个Ver página 9

[43] Chun-Yan L, Zi-Yi Z, Tian-Lin Y, Yi-Li W, Bao L, Jiao L, et al. Liquid biopsy biomarkers of renal interstitial fibrosis based on urinary exosome. Exp Mol Pathol. 2018;105(2):223-8. https:/dx.doi.org/10.1016/j.yexmp.2018.08.004 个Ver página

[44] Solé C, Cortés-Hernández J, Felip ML, Vidal M, Ordi-Ros J. miR-29c in urinary exosomes as predictor of early renal fibrosis in lupus nephritis. Nephrol Dial Transplant. 2015;30(9):1488-96. https://dx.doi.org/10.1093/ndt/gfv128 个Ver página 9 
[45] Garcia-Vives E, Solé C, Moliné T, Vidal M, Agraz I, Ordi-Ros J, et al. The Urinary Exosomal miRNA Expression Profile is Predictive of Clinical Response in Lupus Nephritis. Int J Mol Sci. 2020;21(4):1372. https:/dx.doi.org/10.3390/ijms21041372 $\uparrow$ Ver página 9

[46] Solé C, Moliné T, Vidal M, Ordi-Ros J, Cortés-Hernández J. An Exosomal Urinary miRNA Signature for Early Diagnosis of Renal Fibrosis in Lupus Nephritis. Cells. 2019;8(8):773. https://x.doi.org/10.3390/cells8080773 $\uparrow$ Ver página 9, 10

[47] Li Y, Xu X, Tang X, Bian X, Shen B, Zhao H, et al. MicroRNA expression profile of urinary exosomes in Type IV lupus nephritis complicated by cellular crescent. J Biol Res (Thessalon). 2018;25(1):16. https:/dx.doi.org/10.1186/s40709-018-0088-0 $\uparrow$ Ver página 9

[48] Guan J, Wang G, Tam LS, Kwan BCH, Li EKM, Chow KM, et al. Urinary sediment ICAM-1 level in lupus nephritis. Lupus. 2012;21(11):1190-5. https://dx.doi.org/10.1177/ $0961203312451334 \uparrow$ Ver página 9, 10

[49] Jakiela B, Kosałka J, Plutecka H, Węgrzyn AS, Bazan-Socha S, Sanak M, et al. Urinary cytokines and mRNA expression as biomarkers of disease activity in lupus nephritis. Lupus. 2018;27(8):1259-70. https:/dx.doi.org/10.1177/0961203318770006 个Ver página 9

[50] Perez-Hernandez J, Forner MJ, Pinto C, Chaves FJ, Cortes R, Redon J. Increased Urinary Exosomal MicroRNAs in Patients with Systemic Lupus Erythematosus. PLoS One. 2015;10(9):e0138618. https://dx.doi.org/10.1371/journal.pone.0138618 个Ver página 10 